\title{
Internet of Things Based Indoor Cooling System Using Measurement of Human Body Temperature
}

\author{
R. R. Mohamed ${ }^{1, *}$, M.A. Mohamed ${ }^{2}$, Arniyati Ahmad ${ }^{3}$, Wahidah Hashim ${ }^{1}$, \\ Ahmad Faris Harun Iskandar ${ }^{1}$ \\ ${ }^{1}$ College of Computer Science and Information Technology, Universiti Tenaga \\ Nasional, Malaysia \\ ${ }^{2}$ Faculty of Informatics and Computing, Universiti Sultan Zainal Abidin, Besut, \\ Terengganu, Malaysia \\ ${ }^{3}$ Faculty Science and Technology Defence, Universiti Pertahanan Malaysia, Sg. \\ Besi Camp, Kuala Lumpur, Malaysia \\ "rajina@uniten.edu.my
}

\begin{abstract}
IOT can increase process efficiency while reducing operating costs through smart and intelligent system as well as reducing asset utilization, i.e. energy. Given the current shortage of energy resources and their use proportional exponent, then there must be a way of "smart" to optimize its cost. Air conditioning system for example, requires a smart cooling that can give optimum comfort to the user but at the same time save energy costs. Adjusting a room air conditioner by considering human's temperature and their comfort level is more energy efficient. This is due to the level of comfort and individual body temperature are different from each other. Surrounding temperature either hot or cold perhaps will not give the same effect to individuals, thus a prototype that can control air condition based on human body temperature and room temperature is developed. This prototype is to control the air conditioning based on the human body temperature as well as surrounding temperature. This prototype using a programmable Digital Thermometer which capable to measure room temperature and human body temperature. Reading from each individuals and surrounding will be recorded, calculated and average value will be taken in order to control the room air conditioner without the need for users to adjust manually. This prototype development can improve the efficiency of energy use towards green environment.
\end{abstract}

Keywords: Cooling system, human body temperature, energy saving, equal comfort

\section{Introduction}

Environmental sustainability is a matter that should be given serious attention towards enhancing the stability of the environment. The efficiency of energy consumption must be addressed accordingly as it is one of the largest contributors to the environment through the burning of fuels and electric power generation. Energy savings towards green environment that can contribute to the comfort and safety can be realized through a smart management tools and devices [1,2]. Smart energy management method is discussed in many previous studies $[3,4,5]$. Air conditioning and ventilation systems for example require a smart cooling system that can give optimum comfort to the user while at the same time save energy costs. However, the existing prototype for air conditioning system do not consider the comfort level of the current user's body. Moreover, the comfort level of each individual is different from one person to another and is influenced by several factors such as ambient temperature. Individual under hot weather would require more coolness than individual in a cool place. Human body parts are also different in coldness 
level. Figure 1 shows a relationship between ambient temperature with human body parts (hands, feet, head, upper body as well as the middle part of our body) $[6,7,8]$.

Looking at this situation, a prototype is established that can auto adjust the temperature in the building, which also consider the comfort level of the human body. This prototype will function in such a way that different individual's body temperature is taken into account in order to control / adjust the room temperature. So if there are many individuals who experience different situations, then one value will be taken as an average for temperature adjustment. Hence, everyone will enjoy the temperature of the cooling air equally and fair. Furthermore, the room temperature will be adjusted automatically by also taking into account the room temperature as the environment. This is because the room temperature is also affected by the weather outside, the number of users and the number of electric devices (heat sources) contained in a building. Through this method, electric energy can be saved because the aircond temperature is adjusted according to the needs of people rather than having a fixed temperature value for a period of time.

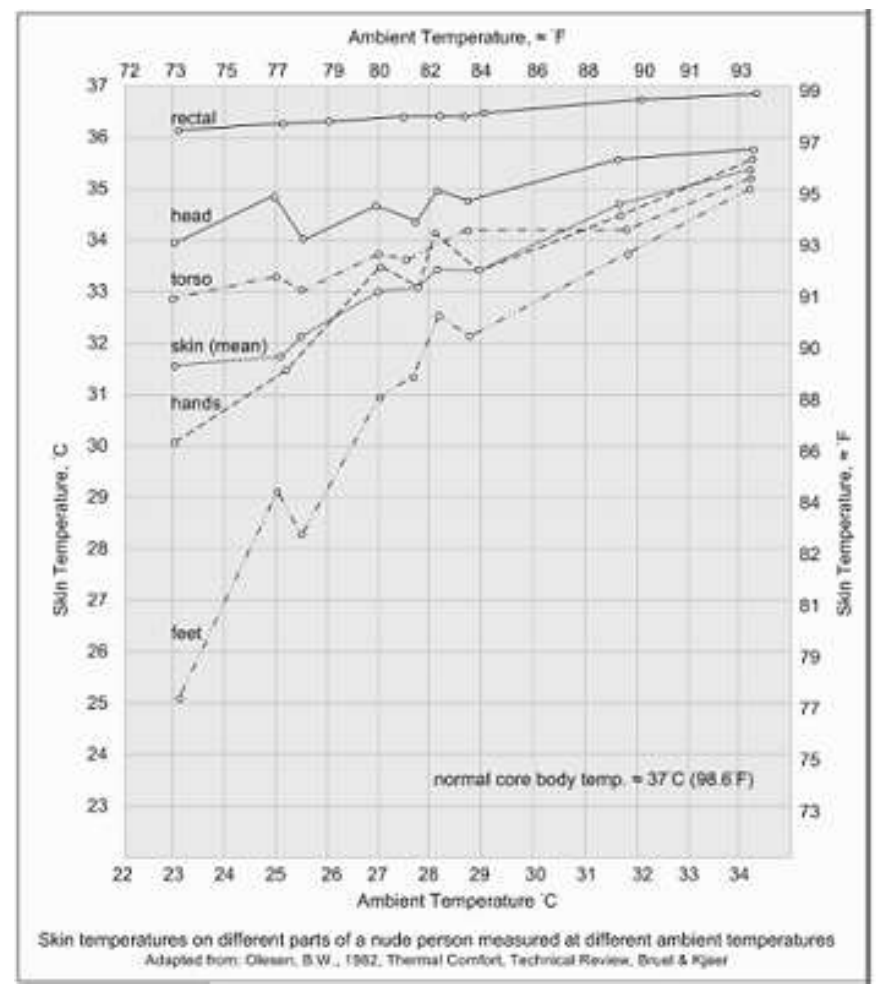

Figure 1. A Relation between Human Body Temperature and Ambient Temperature

\section{Related Works}

In [10] paper proposed the calculation method as an energy saving for indoor air conditioning. Number of people it is calculated using a few modules to control air conditioner system based on how many people were in the building at the time. Result obtained showed that this methods are effective in saving energy for temperature control air-conditioner. While in [11], has proposed a method to detect the behavior of a vacancy for a building through temperature. Through the approach of based learning and neural network, the proposal can detect the number of people in a room based on the room temperature as well as other information related to a heat source. In [12] have developed a system that saves energy and lighting control system according to individual distance on their desk in an office. In other words, 
lighting and air conditioner will be on if there is human existence in the area near their table. This system has been assessed with some real measurement and simulation. Result obtained from test done shows the effectiveness of energy saving method to save energy.

\section{Prototype Description}

\subsection{Prototype Scenario}

A person A just came back from outside while the temperature is too hot. When he arrived home, he has to adjust the remote control of the home air-condition according to his current condition. If after a period of time he may already feel comfortable, then A, needs to lower the temperature of the air conditioner. On the contrary, his children does not really feel the same as he does, so, A has to keep on adjusting the temperature to suit the occupant at that time. Thus this prototype will be introduced to overcome the various problems faced by the residents of the house. The system works like the picture in Figure 2. A cold and hot temperature from different individual will be taken, send to the server for some calculation and adjustment and send an instruction to the air-conditioner to auto adjust.

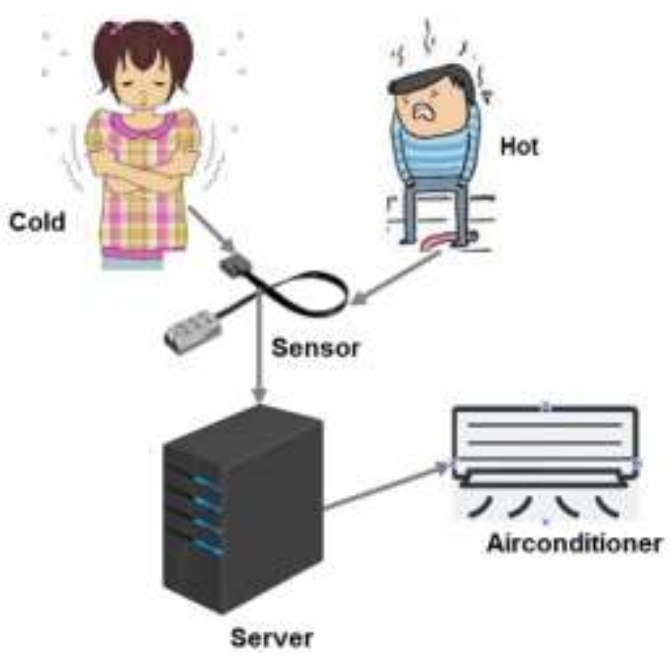

Figure 2. Hot and Cold Scenario

\section{Prototype Description}

\subsection{Software and Hardware Requirements}

Arduino NANO a microcontroller is selected because it provides facilities such as able to communicate with the computer, and programmable with Arduino software. Programmable Resolution 1-wire Digital Thermometer (DS18B20) is a sensor that is selected for measuring body temperature. It is simple to use and also can be benefitted for HVAC environmental control, temperature monitoring systems inside buildings and in a monitoring and controlling systems. A mini fan is used to replicate an air conditioner temperature adjustment. The fan speed can be adjusted to high, low, or medium speed based on the input from Thermopile sensor. To transmit and receive data between components, Radio Frequency (RF) transmitter module communication protocol is used with 9 Volt battery power adapter to supply electric power to all electronic components. Arduino Platform Development tool is used as a software to program the microcontroller in our prototype. It is a compatible product because it features intuitive IDE with smart compiler and advanced optimization. 


\subsection{Prototype Design}

The Digital Thermometer Sensor (DS18b20), Fan, Radio Transmitter (RF) connected on board so that each components can functioned respectively. This project is divided into two parts, which is transmitter and receiver. Transmitter which is attached with the Digital Thermometer Sensor will read the temperature. The data of the room or body temperature will be sent into receiver part. Receiver part will receive the data from the sensor and will control the speed of the fan accordingly. Figure 3 shows the project architecture using the related components.

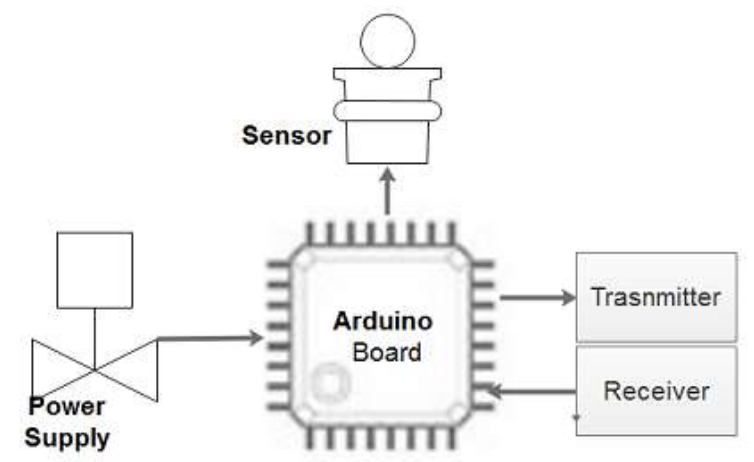

\section{Figure 3. System Architecture}

Figure 4 and Figure 5 show the real setup of the prototype that contains the hardware components as explained earlier. This prototype is taking into account the current condition of the human body temperature. Cold and heat readings felt by humans will be sensed by the temperature sensor. The sensor then sends the readings to the system controller to instruct fan to increase or decrease speed. Figure 6 shows a flowchart process for the prototype implementation.

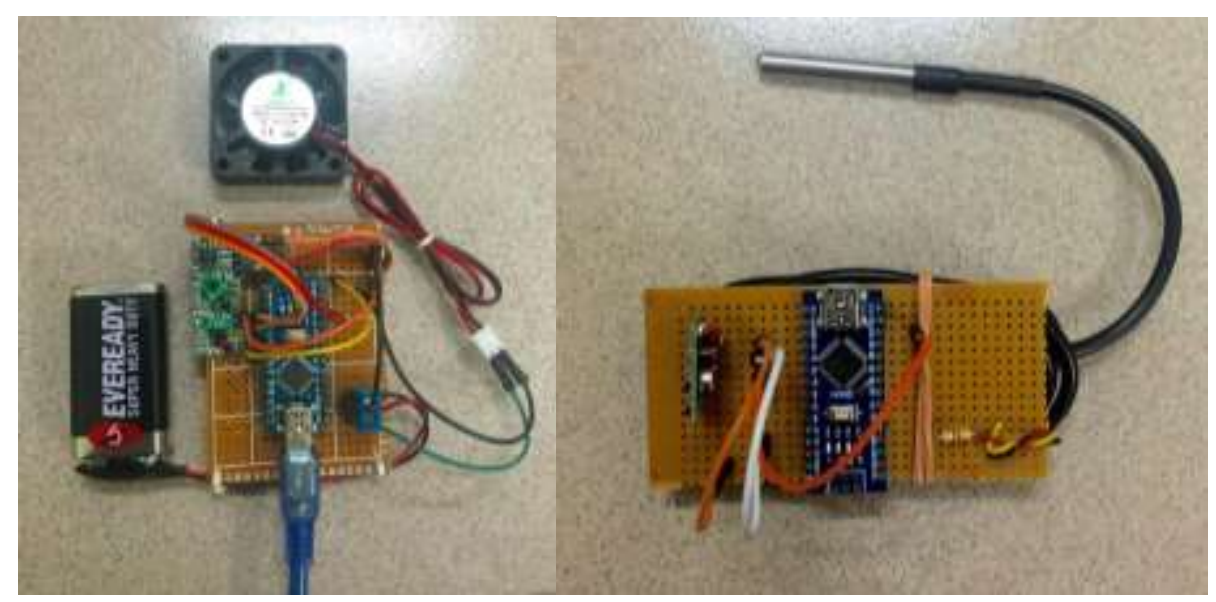

Figure 4 and Figure 5. Digital Sensor and Fan attached to the Arduino Board 


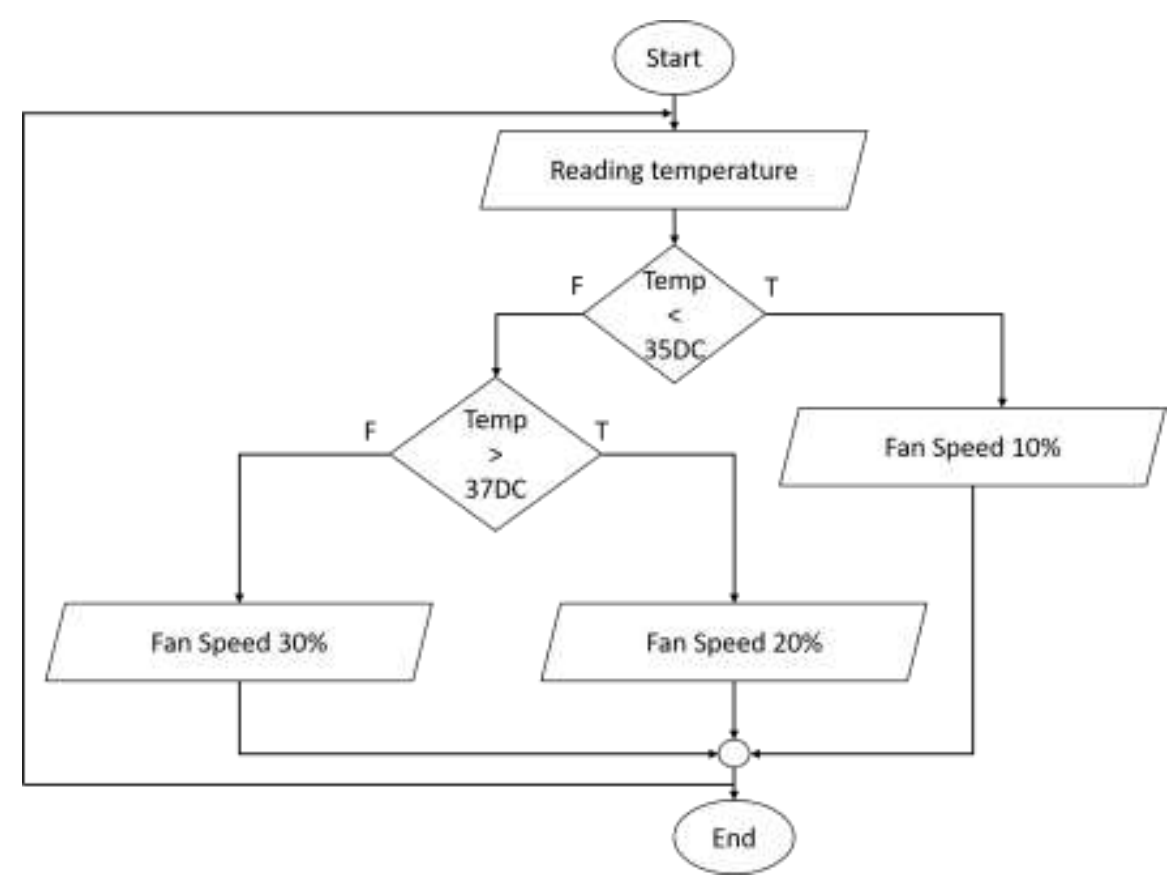

Figure 6. System Flowchart

The transmitter device will read the temperature by using Programmable Resolution 1-Wire Digital Thermometer (DS18B20) sensor. Then the board will calculate the data, if the temperature is lesser than 35 degrees Celsius then the controller will control the fan speed in first condition which is $10 \%$ speed. Else if the temperature is higher than 35 degree Celsius and lower than 37 degrees Celsius, the controller will set the fan speed at the condition 2 which is $20 \%$ speed. Then if the temperature is higher than 37 degrees Celsius, the fan speed will increase to $30 \%$.

\subsection{Prototype Testing}

The threshold value was measured through a random reading from users from $8 \mathrm{am}$ until $6 \mathrm{pm}$ to get feedback for the connection between room temperature and comfort level. The Table 1 below shows number of users and related reading of room temperature. Based on these observations as shown in Table 1, the threshold is set at 37 degree Celsius for discomfort and 23 degree Celsius for comfort threshold.

Table 1. Temperature and Relation of Comfort from the Random Respondents

\begin{tabular}{|l|l|l|}
\hline User & Room temperature & Status \\
\hline 1 & 23 & Comfort \\
\hline 2 & 36 & Not comfort \\
\hline 3 & 39 & Not comfort \\
\hline 4 & 25 & Comfort \\
\hline 5 & 27 & Less comfort \\
\hline 6 & 37 & Not comfort \\
\hline 7 & 36 & Not comfort \\
\hline
\end{tabular}

A few users were randomly selected for the testing of the prototype capabilities. Two types of testing has been conducted. The first testing is to test the ability of this prototype to measure human body's temperature and the second testing is to read room temperature. 
Another testing is carried out by taking the current room temperature. For the human body temperature, the reading captured by the Digital Thermo Sensor was validated by the normal Thermometer. The readings taken were compared as shown in Figure 7. While room temperature reading was validated by comparing that temperature with an online weather forecast as shown by Figure 7.

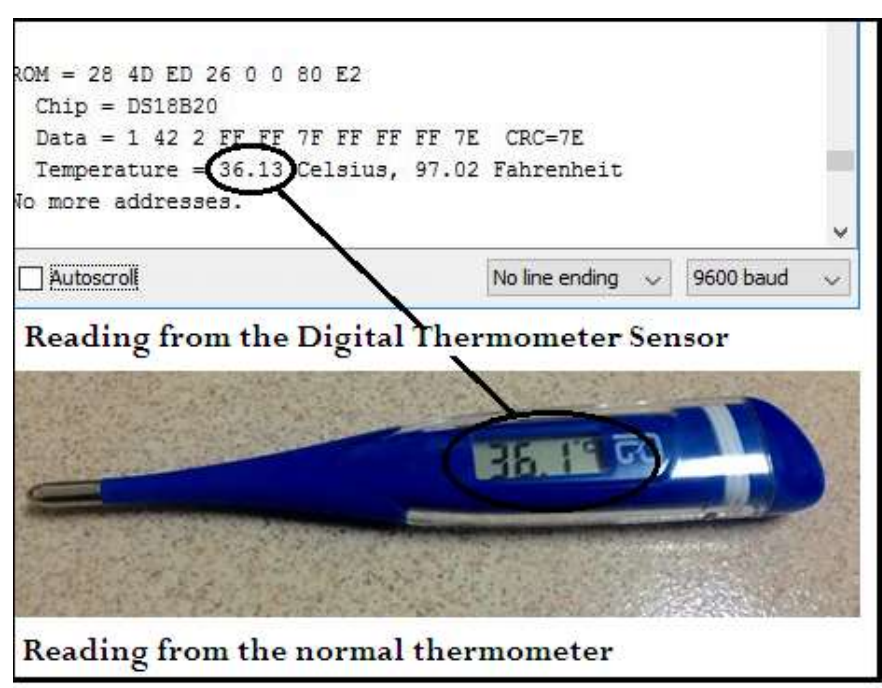

Figure 7. Reading Comparison between Two Types of Thermometer

\subsection{Reliability Testing}

Testing conducted involving a10 users under 2 conditions. 10 users coming out from the warm/hot place, and 10 new users out of a cold place. For this testing, two of thermometers are used to measure two different temperatures at the same time under the two different scenarios.

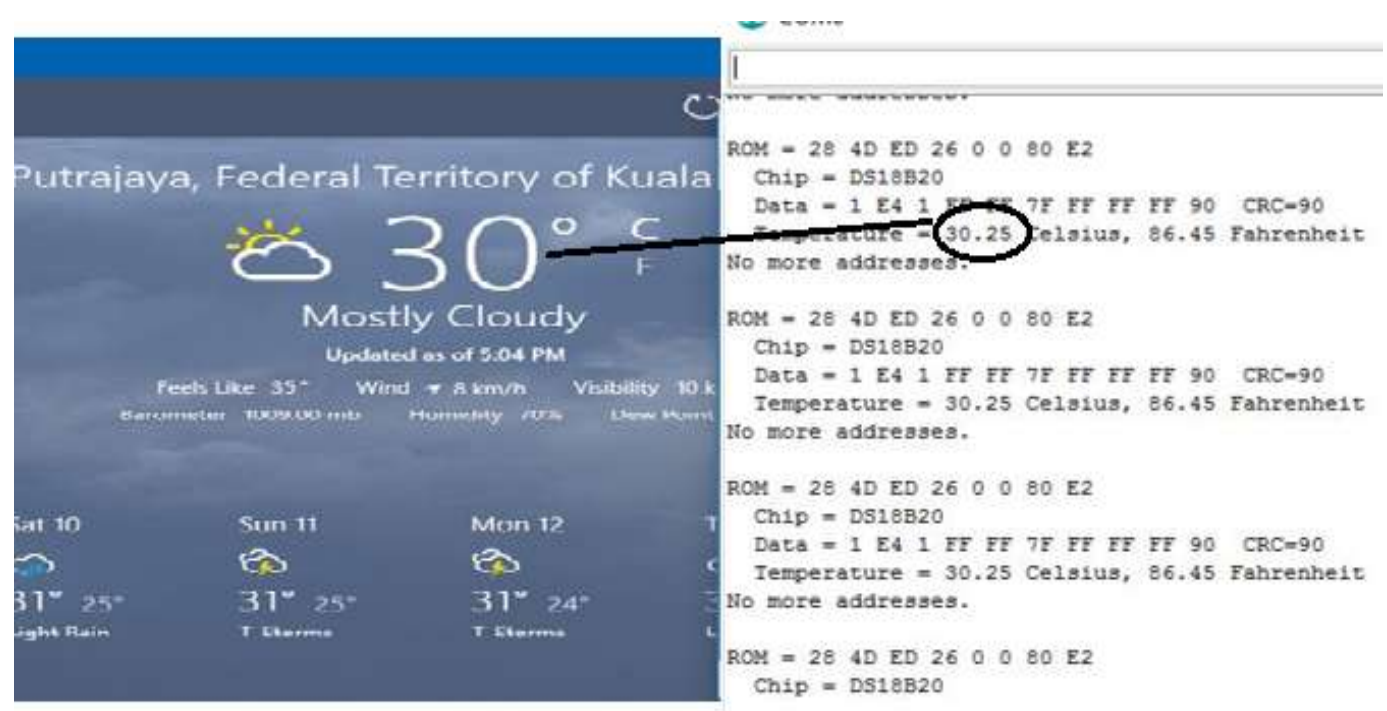

Figure 8. Room Temperature Comparison

\section{Result and Discussion}

Table 2 shows a list of temperatures from 50 users based on the conditions described earlier. We scale users with ratio 1:10. Both readings taken were compared to a normal ordinary thermometer to test the validity of the readings. From the table, average 
temperature of humans in the hot area is 36, while in cold area is 23 . Different temperature among individual is dependent upon their physical traits such as genetics, age, height, obesity [9]. Based on this temperature, speed will be adjusted accordingly to the average value formulated in (1).

$$
\mathrm{AVT}=\frac{1}{n} \sum_{i=1}^{n} a_{i}=\frac{1}{n}\left(a_{1}+a_{2}+\cdots+a_{n}\right)
$$

where AVT, $\mathrm{n}$ and a are the average temperature, number of temperatures, and value for each temperature respectively. Measured result is tabulated in Table 2. While graph is as shown in Figure 8 plotted by the tabulated data in Table 2.

Table 2 - Temperature Measured and Average

\begin{tabular}{|l|l|l|l|l|}
\hline \multicolumn{2}{|c|}{ From the hot area } & \multicolumn{2}{c|}{ Form the cold } & \\
\hline $\begin{array}{l}\text { Digital } \\
\text { Thermometer }\end{array}$ & $\begin{array}{l}\text { Normal } \\
\text { Thermometer }\end{array}$ & $\begin{array}{l}\text { Digital } \\
\text { Thermometer }\end{array}$ & $\begin{array}{l}\text { Normal } \\
\text { Thermometer }\end{array}$ & $\begin{array}{l}\text { Average } \\
\text { Temperature }\end{array}$ \\
\hline 37.1 & 37 & 27 & 26.9 & 32.05 \\
\hline 36 & 36.1 & 25 & 25.1 & 30.5 \\
\hline 36 & 36.9 & 23 & 23 & 29.5 \\
\hline 37.2 & 37.2 & 23 & 23.1 & 30.1 \\
\hline 38 & 37.9 & 18 & 18.2 & 28 \\
\hline 35 & 35.1 & 24 & 24 & 29.5 \\
\hline
\end{tabular}

Graph in Figure 9 shows the number of temperatures measured and the average temperature. In this case, air-condition temperature will be auto adjusted based on this average readings.

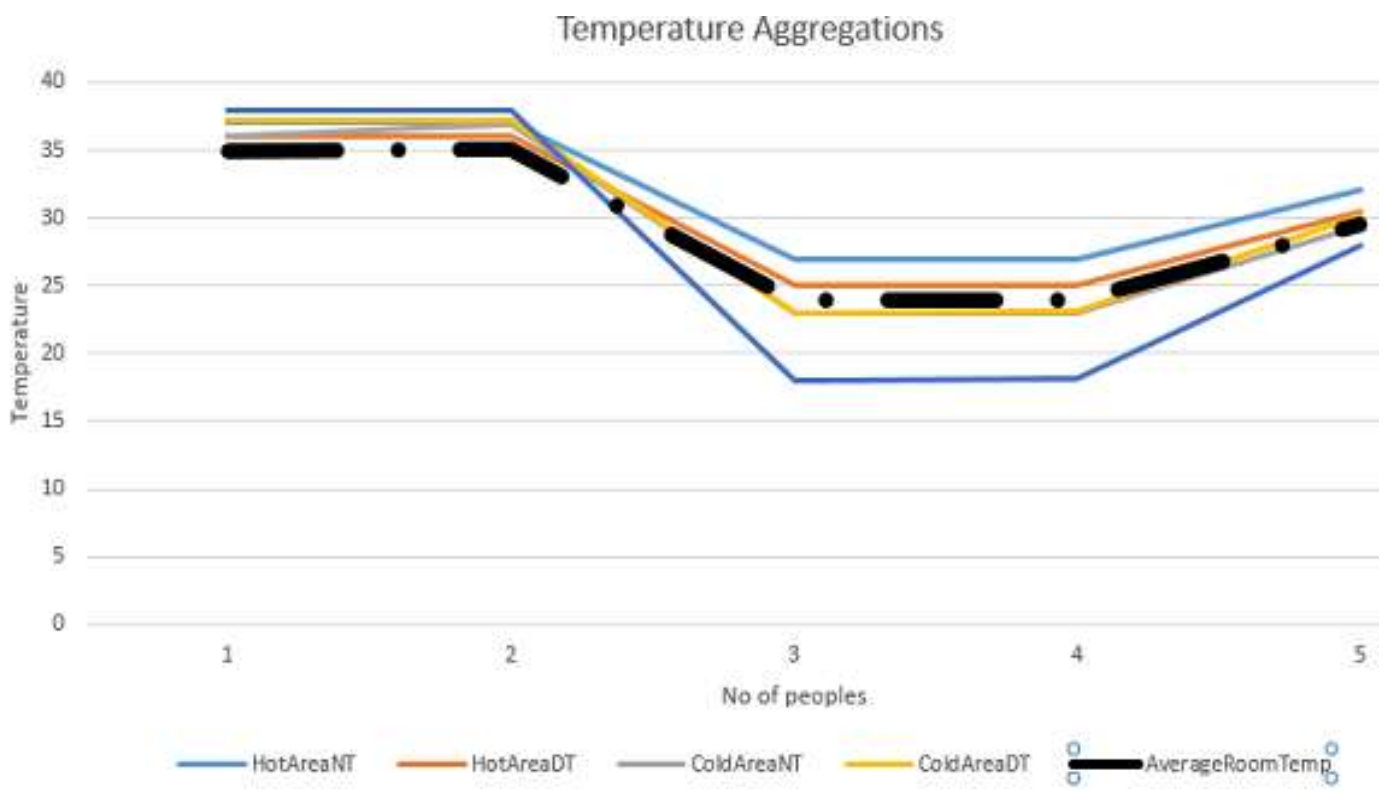

Figure 9. Graph Plotted for Temperature Aggregations 


\section{Conclusion}

The study of temperature control aircond based on aggregate human body temperature have been carried out. Human body temperature readings are recorded using sensors from two different areas of cold and heat. Both readings were taken and the average temperature of the aircond adjusted average. Subsequent studies involving more than two data acquisition thermometer and should be taken from a variety of cold and hot conditions to see the reliability of the prototype.

\section{Acknowledgements}

This project funded by UNITEN.

\section{References}

[1] M. Casini, "Internet of things for Energy efficiency of buildings', ICAEE 2014, NICE.

[2] Optimizing Building Energy Use, http://www.achrnews.com/articles/128876-optimizing-buildingenergy-use

[3] M. Mercy Sharon, "Towards the Implementation of IoT for Environmental Condition Monitoring in Homes", www. Academia. Edu.

[4] F. A. Barata, R. N. Silva, "Distributed Model Predictive Control for Housing with Hourly Auction of Available Energy", Springer Link, 4th IFIP WG 5.5/SOCOLNET Doctoral Conference on Computing, Electrical and Industrial Systems, DoCEIS 2013, Costa de Caparica, Portugal, (2013).

[5] Mukesh Taneja, "A framework for power saving in IoT networks", Advances in Computing, Communications and Informatics (ICACCI, 2014 International Conference on

[6] "Facts About Your Skin - Temperatures in and on the human body", http://www.healthyheating.com/Definitions/facts_about_skin.htm\#.WBaw4dJ94ps

[7] Fabbri, Kristian, "Indoor Thermal Concept Perception - A Brief History of Thermal Comfort: From Effective Temperature to Adaptive Thermal Comfort", Springer, (2015).

[8] J Haby, "The 6 factors that Influence Human Outside Comfort", online access, http://www.theweatherprediction.com/habyhints/36/.

[9] Health and Safetty Executive - The basic factors", http://www.hse.gov.uk/temperature/thermal/ factors.htm

[10] Q. Ye, Z. Yang, "A method of automatic people counting used in air-conditioning energy-saving", ICCET (2010).

[11] H. Zhao, Hengyang Zhao; Zhongdong Qi; Shujuan Wang; Kambiz Vafai; Hai Wang; Haibao Chen Sheldon X.-D. Tan, "Learning-based occupancy behavior detection for smart buildings", ISCAS, (2016)

[12] Y. Kaneko, M. Matsushita1, S. Kitagami, J. Sawamoto, T. Shiotsuki, T. Munaka, “An Energy-Saving Control System of Lighting and Air Conditioning Linked to Employee's Entry/Exist in the Zone of the Office", International Journal of Energy Engineering, vol. 5, no. 4, pp. 67-73. 